\title{
Close Form Solutions of the Fractional Generalized Reaction Duffing Model and the Density Dependent Fractional Diffusion Reaction Equation
}

\author{
M. Hafiz Uddin ${ }^{1}$, M. Ali Akbar², Md. Ashrafuzzaman Khan², Md. Abdul Haque ${ }^{2}$ \\ ${ }^{1}$ Department of Mathematics, Jessore University of Science and Technology, Jessore, Bangladesh \\ ${ }^{2}$ Department of Applied Mathematics, University of Rajshahi, Rajshahi, Bangladesh
}

Email address:

ali_math74@yahoo.com (M. A. Akbar)

To cite this article:

M. Hafiz Uddin, M. Ali Akbar, Md. Ashrafuzzaman Khan, Md. Abdul Haque. Close Form Solutions of the Fractional Generalized Reaction Duffing Model and the Density Dependent Fractional Diffusion Reaction Equation. Applied and Computational Mathematics.

Vol. 6, No. 4, 2017, pp. 177-184. doi: 10.11648/j.acm.20170604.13

Received: March 21, 2017; Accepted: April 5, 2017; Published: July 14, 2017

\begin{abstract}
The two variable $\left(G^{\prime} / G, 1 / G\right)$-expansion method is significant for finding the exact traveling wave solution to nonlinear evolution equations (NLEEs) in mathematical physics, applied mathematics and engineering. In this article, we exert the two variable $\left(G^{\prime} / G, 1 / G\right)$-expansion method for investigating the fractional generalized reaction Duffing model and density dependent fractional diffusion reaction equation and obtain exact solutions containing parameters. When the parameters are taken particular values, traveling wave solutions are transferred into the solitary wave solutions. The two variable $\left(G^{\prime} / G, 1 / G\right)$-expansion method is the generalization of the original $\left(G^{\prime} / G\right)$-expansion method established by Wang et al [21].
\end{abstract}

Keywords: Nonlinear Evolution Equation, Fractional Generalized Reaction Duffing Model, Density Dependent Fractional Diffusion Equation, Traveling Wave Solution

\section{Introduction}

The significance of nonlinear evolution equations is now well established. In the last three decades nonlinear phenomena are one of the most impressive fields of research. Nonlinear phenomena occur in various branches of science, engineering and biology, such as fluid mechanics, plasma physics, solid state physics, optical fiber, gas dynamics, elasticity, biomechanics, relativity, ecology, biophysics and so on. Since the appearance of solitary wave in natural science is expanding day by day, it is important to find the solitary wave solutions to NLEEs. The exact solutions to NLEEs help us to provide information about the structure of complex phenomena. As a key problem, finding their exact solutions is of great importance and it is actually executed through various efficient and powerful method, such as, the Hirota method [1], the Backlund transform method [2,3], the inverse scattering transform method [4], the Jacobi elliptic function expansion method [5-7], the truncated Painleve expansion method [8-11], the tanh function method [12-15], the Exp-function method [16-22], the $\left(G^{\prime} / G\right)$-expansion method [23-30], the improved $\left(G^{\prime} / G\right)$-expansion method [31-32], the two variable $\left(G^{\prime} / G, 1 / G\right)$-expansion method $[33,34]$, the first integral method [35] etc. The main concept of the $\left(G^{\prime} / G\right)$-expansion method is the exact solution of nonlinear NLEEs are revealed by a polynomial in one variable $\left(G^{\prime} / G\right)$ in which $G=G(\xi)$ satisfies the second order ordinary differential equation (ODE) $G^{\prime \prime}(\xi)+$ $\lambda G^{\prime}(\xi)+\mu G(\xi)=0$, where $\lambda$ and $\mu$ are constants. In this article, we use the two variable $\left(G^{\prime} / G, 1 / G\right)$-expansion method, which is the general case of the $\left(G^{\prime} / G\right)$-expansion method. The main concept of the two variable $\left(G^{\prime} / G, 1 / G\right)$ expansion method is the exact traveling wave solutions of nonlinear NLEEs can be written as a polynomials in two variables $\left(G^{\prime} / G\right)$ and $(1 / G)$, in which $G=G(\xi)$ satisfies a second order linear $\operatorname{ODE} G^{\prime \prime}(\xi)+\lambda G(\xi)=\mu$, where $\lambda$ and $\mu$ are constants. The degree of the polynomial can be evaluated by taking homogeneous balance between the highest-order derivatives and nonlinear terms in the given nonlinear PDEs, where the coefficient of the polynomial can be determined by solving a set of algebraic equations. Recently, Li et al [33] 
and Zayed et al [34] applied the two variable $\left(G^{\prime} / G, 1 / G\right)$ expansion method and determined the exact solution of nonlinear NLEEs.

The objective of this article is study the fractional generalized reaction Duffing model and density dependent fractional diffusion reaction equation by making use of the two variable $\left(G^{\prime} / G, 1 / G\right)$-expansion method.

\section{Description of the Two Variable $\left(G^{\prime} / G, 1 / G\right)$-expansion Method}

Before starting the description of the $\left(G^{\prime} / G, 1 / G\right)$ expansion method [33, 34], we discuss about the following fundamental concepts. Let us consider the second order ordinary differential equation (ODE):

$$
G^{\prime \prime}(\xi)+\lambda G(\xi)=\mu,
$$

where, $\varphi=\left(G^{\prime} / G\right)$ and $\psi=1 / G$, then we obtain

$$
\varphi^{\prime}=-\varphi^{2}+\mu \psi-\lambda \psi^{\prime}=-\varphi \psi .
$$

Remark 1: If $\lambda<0$, the general solution of equation (1) is:

$$
G(\xi)=A_{1} \sinh (\sqrt{-\lambda} \xi)+A_{2} \cosh (\sqrt{-\lambda} \xi)+\frac{\mu}{\lambda}
$$

where $A_{1}$ and $A_{2}$ are arbitrary constants.

Consequently, we obtain

$$
\psi^{2}=\frac{-\lambda}{\lambda^{2} \sigma+\mu^{2}}\left(\varphi^{2}-2 \mu \psi+\lambda\right)
$$

where $\sigma=A_{1}{ }^{2}-A_{2}{ }^{2}$.

Remark 2: If $\lambda>0$, the general solution of equation (1) is:

$$
G(\xi)=A_{1} \sin (\sqrt{\lambda} \xi)+A_{2} \cos \sqrt{\lambda} \xi+\frac{\mu}{\lambda}
$$

where $A_{1}$ and $A_{2}$ are arbitrary constants. Consequently, we obtain

$$
\psi^{2}=\frac{\lambda}{\lambda^{2} \sigma-\mu^{2}}\left(\varphi^{2}-2 \mu \psi+\lambda\right)
$$

where $\sigma=A_{1}{ }^{2}-A_{2}{ }^{2}$.

Remark 3: If $\lambda=0$, the general solution of equation (1) is:

$$
G(\xi)=\frac{\mu}{2} \xi^{2}+A_{1} \xi+A_{2}
$$

where $A_{1}$ and $A_{2}$ are arbitrary constants and hence

$$
\begin{aligned}
& \qquad \psi^{2}=\frac{1}{A_{1}{ }^{2}-2 \mu A_{2}}\left(\varphi^{2}-2 \mu \psi\right) \\
& \qquad \frac{\partial^{2 \alpha} U(x, t)}{\partial t^{2 \alpha}}+p \frac{\partial^{2 \alpha} U(x, t)}{\partial t^{2 \alpha}} \\
& \text { where } t>0,0<\alpha \leq 1 \text {, here } p, q, r \text { and } s \text { are consta } \\
& \text { we take } r=0 \text {, then Eq. (12) converts into } \\
& \frac{\partial^{2 \alpha} U(x, t)}{\partial t^{2 \alpha}}+p \frac{\partial^{2 \alpha} U(x, t)}{\partial t^{2 \alpha}} q U(x, t)+s U^{3}(x, t)=0
\end{aligned}
$$

Assume the nonlinear partial differential equation is in the form

$$
Q=\left(U, U_{z}, U_{x}, U_{x x}, U_{x z}, U_{z z}, \cdots\right)
$$

where $U=U(x, t)$ is an unknown function and $Q$ is a polynomial of $U(x, t)$ and its partial derivative.

Step 1: Consider the traveling wave transformation

$$
\xi=x-v t, U(x, t)=U(\xi),
$$

where $v$ is the speed of traveling wave.

The wave variable permits us to reduce equation (9) into an ODE for $U=U(\xi)$ :

$$
R=\left(U, U^{\prime}, U^{\prime \prime}, U^{\prime \prime \prime}, \ldots\right)=0
$$

where $R$ is a polynomial of $U(\xi)$ and its total derivative with respect to $\xi$.

Step 2: Assume that the solution of equation (10) can be written as a polynomial in two variables $\varphi$ and $\psi$ as follows:

$$
U(\xi)=\sum_{i=0}^{N} a_{i} \varphi^{i}+\sum_{i=1}^{N} b_{i} \varphi^{i-1} \psi
$$

where, $a_{i}(i=0,1, \ldots, N)$ and $b_{i}(i=0,1, \ldots, N)$ are constant to be determined later.

Step 3: Taking homogeneous balance between the highest order derivatives and the nonlinear terms appearing in equation (10) to determine the positive integer $N$ in equation (11).

Step 4: Substitute equation (11) into equation (10) along with (2) and (4), the equation (10) can be reduce into a polynomial in $\varphi$ and $\psi$, in which the degree of $\psi$ is no longer than one. Equating the coefficients of this polynomial of like power to zero gives a system of algebraic equations which can be solved by using the software Maple or Mathematica to get the values of $a_{i}, b_{i}, v, \mu, A_{1}, A_{2}$ and $\lambda$ where $\lambda<0$.

Step 5: Similar application to step 4, substitute equation (11) into equation (10) along with (2) and (6) for $\lambda>0$ (or (2) and (8) for $\lambda=0$ ), we attain the exact solutions of equation (10) represented by trigonometric functions (or by rational function respectively).

\section{Application}

\subsection{The Fractional Generalized Duffing Model}

In this subsection, we apply the two variables $\left(G^{\prime} / G, 1 / G\right)$-expansion method to obtain exact solutions of fractional generalized Duffing model which is in the form: where $t>0,0<\alpha \leq 1$, here $p, q, r$ and $s$ are constants. If

where $t>0,0<\alpha \leq 1$.

Now we introduce the fractional wave transformation:

$$
\xi=\frac{K x^{\alpha}}{\Gamma(1+\alpha)}-\frac{C t^{\alpha}}{\Gamma(1+\alpha)}
$$

where $C$ and $K$ are non-zero constants.

Using the transformation (14), Eq. (12) reduced into the following ODE for $U=U(\xi)$ :

$$
\left(C^{2}+P k^{2}\right) U^{\prime \prime}+q U+s U^{3}=0
$$


Balancing the highest order derivative $U^{\prime \prime}$ and nonlinear term $U^{3}$, yields $N=1$.

Therefore the solution (11) is of the following form:

$$
\begin{aligned}
& U(\xi)=a_{0}+a_{1} \varphi(\xi)+b_{1} \psi(\xi) \\
& \varphi^{0}: 2 s b_{1}^{3} \lambda^{3} \mu-q a_{0} \lambda^{4} \sigma^{2}-c^{2} b_{1} \lambda^{2} \mu^{3}-s a_{0}^{3} \lambda^{4} \sigma^{2}+3 s a_{0} b_{1}^{2} \lambda^{2} \mu^{2}-s a_{0}^{3} \mu^{4}-c^{2} b_{1} \lambda^{4} \mu \sigma-2 s a_{0}^{3} \mu^{2} \sigma \lambda^{2}-2 q a_{0} \mu^{2} \lambda^{2} \sigma \\
& -p k^{2} b_{1} \lambda^{2} \mu^{3}-q a_{0} \mu^{4}+3 s a_{0} b_{1}^{2} \lambda^{4} \sigma-p k^{2} b_{1} \lambda^{4} \mu \sigma=0 \\
& \varphi^{1}:-q a_{1} \lambda^{4} \sigma^{2}-2 p k^{2} a_{1} \lambda \mu^{4}-6 s a_{0}^{2} a_{1} \lambda^{2} \sigma \mu^{2}+3 s a_{1} b_{1}^{2} \lambda^{2} \mu^{2}-4 p k^{2} a_{1} \lambda^{3} \sigma \mu^{2}-q a_{1} \mu^{4}-3 s a_{0}^{1} a_{1} \mu^{4} \sigma^{2}-2 c^{2} a_{1} \lambda^{5} \sigma^{2} \\
& -2 c^{2} a_{1} \lambda \mu^{4}-2 p k^{2} a_{1} \lambda^{5} \sigma^{2}-3 s a_{0}^{1} a_{1} \mu^{4}-2 q a_{1} \mu^{2} \lambda^{2} \sigma+3 s a_{1} b_{1}^{2} \lambda^{4} \sigma-4 c^{2} a_{1} \lambda^{3} \sigma \mu^{2}=0 \\
& \varphi^{2}:-c^{2} b_{1} \lambda^{3} \mu \sigma-p k^{2} b_{1} \lambda^{3} \mu \sigma-3 s a_{0} a_{1}^{2} \lambda^{4} \sigma^{2}+3 s a_{0} b_{1}^{2} \lambda^{3} \sigma+2 s b_{1}^{3} \lambda^{2} \mu-6 s a_{0} a_{1}^{2} \lambda^{2} \sigma \mu^{2}-p k^{2} b_{1} \lambda \mu^{3}-3 s a_{0} a_{1}^{2} \mu^{4} \\
& -c^{2} b_{1} \lambda \mu^{3}+3 s a_{0} b_{1}^{2} \lambda \mu^{2}=0 \\
& \varphi^{3}: 3 s a_{1} b_{1}^{2} \lambda \mu^{2}-4 p k^{2} a_{1} \lambda^{2} \sigma \mu^{2}-s a_{1}^{3} \mu^{4}-2 c^{2} a_{1} \mu^{4}-s a_{1}^{3} \lambda^{4} \sigma^{2}-2 p k^{2} a_{1} \lambda^{4} \sigma^{2}+3 s a_{1} b_{1}^{2} \lambda^{3} \sigma-4 c^{2} a_{1} \mu^{2} \lambda^{2} \sigma- \\
& 2 s a_{1}^{3} \mu^{2} \lambda^{2} \sigma-2 p k^{2} a_{1} \mu^{4}-2 c^{2} a_{1} \lambda^{4} \sigma^{2}=0 \\
& \psi: c^{2} b_{1} \lambda \mu^{4}-6 s a_{0}^{2} b_{1} \lambda^{2} \sigma \mu^{2}-3 s a_{0}^{2} b_{1} \mu^{4}-p k^{2} b_{1} \lambda^{5} \sigma^{2}-2 b_{1} \mu^{4}+p k^{2} b_{1} \lambda \mu^{4}-q b_{1} \lambda^{4} \sigma^{2}-c^{2} b_{1} \lambda^{5} \sigma^{2}-2 q b_{1} \mu^{2} \lambda^{2} \sigma \\
& -6 s a_{0} b_{1}^{2} \lambda^{3} \mu \sigma-3 s b_{1}^{3} \lambda^{2} \mu^{2}-6 s a_{0} b_{1}^{2} \lambda \mu^{3}-3 s a_{0}^{2} b_{1} \lambda^{4} \sigma^{2}+s b_{1}^{3} \lambda^{4} \sigma=0 \\
& \varphi \psi: 6 p k^{2} a_{1} \mu^{3} \lambda^{2} \sigma+6 c^{2} a_{1} \mu^{3} \lambda^{2} \sigma-6 s a_{0} a_{1} b_{1} \mu^{4}+3 p k^{2} a_{1} \mu \lambda^{4} \sigma^{2}-12 s a_{0} a_{1} b_{1} \mu^{2} \lambda^{2} \sigma+3 c^{2} a_{1} \mu \lambda^{4} \sigma^{2}+3 p k^{2} a_{1} \mu^{5} \\
& -6 s a_{1} b_{1}^{2} \lambda \mu^{3}-6 s a_{1} b_{1}^{2} \lambda^{3} \mu \sigma-6 s a_{0} a_{1} b_{1} \lambda^{4} \sigma^{2}+3 c^{2} a_{1} \mu^{5}=0
\end{aligned}
$$

Solving the system of algebraic equations in (17) by using symbolic computation software like, Maple or Mathematica, we obtain the following results:

$$
a_{0}=0, a_{1}= \pm \sqrt{\frac{q}{s \lambda}}, b_{1}= \pm \sqrt{\frac{-q \mu^{2}-q \lambda^{2} \sigma}{s}}, k=k, c=\sqrt{\frac{-2 q-p k^{2} \lambda}{\lambda}}
$$

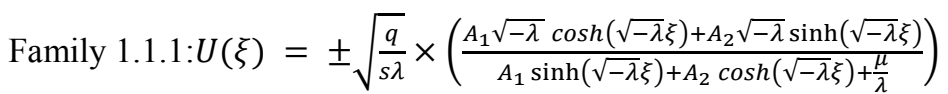

$$
\pm \sqrt{\frac{-q \mu^{2}-q \lambda^{2} \sigma}{s}} \times \frac{1}{A_{1} \sinh (\sqrt{-\lambda} \xi)+A_{2} \cosh (\sqrt{-\lambda} \xi)+\frac{\mu}{\lambda}}
$$

where $\sigma=A_{1}^{2}-A_{2}^{2}$.

Family 1.1.2: If $A_{1}=0, A_{2} \neq 0$ and $\mu=0$ in (18), we obtain the solitary wave solution

$$
U(\xi)= \pm \sqrt{\frac{q}{s \lambda}} \cdot \sqrt{-\lambda} \tanh (\sqrt{-\lambda} \xi) \pm \sqrt{\frac{-q \lambda^{2} \sigma}{s}} \cdot \frac{1}{A_{2}} \operatorname{sech}(\sqrt{-\lambda} \xi)
$$

Family 1.1.3: If $A \neq 0, A=0$ and $\mu=0$, we obtain the solitary wave solution

$$
U(\xi)= \pm \sqrt{\frac{q}{s \lambda}} \sqrt{-\lambda} \operatorname{coth}(\sqrt{-\lambda} \xi) \pm \sqrt{\frac{-q \lambda^{2} \sigma}{s}} \frac{1}{A_{2}} \operatorname{cosech}(\sqrt{-\lambda} \xi)
$$

Case 1.2: For $\lambda>0$, Substituting Eq. (16) into Eq. (15) along with Eq. (2) and Eq. (6) and equating the coefficients to zero yields a set of algebraic equation set of algebraic equations for $a_{0}, a_{1}, b_{1}, \mu, \sigma, \lambda, c, p$ and $k$ as follows:

$$
\begin{gathered}
\varphi^{0}: c^{2} b_{1} \lambda^{4} \mu \sigma-3 s a_{0} b_{1}^{2} \lambda^{4} \sigma+3 s a_{0} b_{1}^{2} \lambda^{2} \mu^{2}+2 s b_{1}^{3} \lambda^{3} \mu-q a_{0} \lambda^{4} \sigma^{2}-c^{2} b_{1} \lambda^{2} \mu^{3}-s a_{0}^{3} \lambda^{4} \sigma^{2}+2 q a_{0} \mu^{2} \lambda^{2} \sigma-s a_{0}^{3} \mu^{4} q a_{0} \mu^{4} \\
+p k^{2} b_{1} \lambda^{4} \mu \sigma-p k^{2} b_{1} \lambda^{2} \mu^{3}+2 s q_{0}^{3} \mu^{2} \lambda^{2} \sigma=0 \\
\varphi^{1}:-q a_{0} \mu^{4}+4 c^{2} a_{0} \lambda^{3} \sigma \mu^{2}-q a_{1} \lambda^{2} \sigma^{2}+3 s a_{1} b_{1}^{2} \lambda^{2} \mu^{2}-2 p k^{2} a_{1} \lambda^{5} \sigma^{2}+4 p k^{2} a_{1} \lambda^{3} \sigma \mu^{2}-3 s a_{0}^{2} a_{1} \mu^{4}+2 q a_{1} \mu^{2} \lambda^{2} \sigma \\
-3 s a_{0}^{2} a_{1} \lambda^{4} \sigma^{2}+6 s a_{0}^{2} a_{1} \mu^{2} \lambda^{2} \sigma-2 p k^{2} a_{1} \lambda \mu^{4}-2 c^{2} a_{1} \lambda \mu^{4}-3 s a_{1} b^{2} \lambda^{2} \sigma-2 c^{2} a_{1} \lambda^{5} \sigma^{2}=0 \\
\varphi^{2}: p k^{2} b_{1} \lambda^{3} \mu \sigma-p k^{2} b_{1} \lambda \mu^{3}+6 s a_{0} a_{1}^{2} \mu^{2} \lambda^{2} \sigma+c^{2} b_{1} \lambda^{2} \mu \sigma-3 s a_{0} b_{1}^{2} \lambda^{3} \sigma-3 s a_{0} a_{1}^{2} \mu^{4}+2 s b_{1}^{3} \lambda^{2} \mu-3 s a_{0} a_{1}^{2} \lambda^{4} \sigma^{2} \\
+3 s a_{0} b_{1}^{2} \lambda \mu^{2}-c^{2} b_{1} \lambda \mu^{3}=0 \\
\quad
\end{gathered}
$$




$$
\begin{gathered}
\varphi^{3}:-s a_{1}^{3} \mu^{4}-2 c^{2} a_{1} \mu^{4}-s a_{1}^{3} \lambda^{4} \sigma^{2}-2 p k^{2} a_{1} \mu^{4}-2 c^{2} a_{1} \lambda^{4} \sigma^{2}-3 s a_{1} b_{1}^{2} \lambda^{2} \sigma+4 p k^{2} a_{1} \mu^{2} \lambda^{2} \sigma-2 p k^{2} a_{1} \lambda^{4} \sigma^{2}+2 s a_{1}^{3} \mu^{2} \lambda^{2} \sigma \\
+4 c^{2} a_{1} \mu^{2} \lambda^{2} \sigma+3 s a_{1} b_{1}^{2} \lambda \mu^{2}=0 \\
\psi: 2 q b_{1} \mu^{2} \lambda^{2} \sigma-p k^{2} b_{1} \lambda^{5} \sigma^{2}-q b_{1} \mu^{4}-3 s a_{0}^{2} b_{1} \mu^{4}-3 s a_{0}^{2} b_{1} \lambda^{4} \sigma^{2}-s b_{1}^{3} \lambda^{4} \sigma-q b_{1} \lambda^{4} \sigma^{2}-6 s a_{0} b_{1}^{2} \lambda \mu^{3}-c^{2} b_{1} \lambda^{5} \sigma^{2} \\
+6 s a_{0}^{2} b_{1} \mu^{2} \lambda^{2} \sigma-3 s b_{1}^{3} \lambda^{2} \mu^{2}+c^{2} b_{1} \lambda \mu^{4}+p k^{2} b_{1} \lambda \mu^{4}+6 s a_{0} b_{1}^{2} \lambda^{3} \mu \sigma=0 \\
\varphi \psi:-6 p k^{2} a_{1} \mu^{3} \lambda^{2} \sigma-6 s a_{0} a_{1} b_{1} \mu^{4}+3 c^{2} a_{1} \mu^{5}-6 c^{2} a_{1} \mu^{3} \lambda^{2} \sigma-6 s a_{1} b_{1}^{2} \lambda \mu^{3}-6 s a_{0} a_{1} b_{1} \lambda^{4} \sigma^{2}+6 s a_{1} b_{1}^{2} \lambda^{3} \mu \sigma \\
+3 p k^{2} a_{1} \mu \lambda^{4} \sigma^{2}+3 p k^{2} a_{1} \mu^{5}+12 s a_{0} a_{1} b_{1} \mu^{2} \lambda^{2} \sigma+3 c^{2} a_{1} \mu \lambda^{4} \sigma^{2}=0 \\
\quad \begin{array}{c}
21) \\
\psi \varphi^{2}:
\end{array} b_{1}^{3} \lambda \mu^{2}-2 c^{2} b_{1} \lambda^{4} \sigma^{2}-s b_{1}^{3} \lambda^{3} \sigma-2 p k^{2} b_{1} \mu^{4}-3 s a_{1}^{2} b_{1} \mu^{4}+4 p k^{2} b_{1} \mu^{2} \lambda^{2} \sigma-2 p k^{2} b_{1} \lambda^{4} \sigma^{2}-3 s a_{1}^{2} b_{1} \lambda^{4} \sigma^{2}+ \\
4 c^{2} b_{1} \mu^{2} \lambda^{2} \sigma-2 c^{2} b_{1} \mu^{4}+6 s a_{1}^{2} b_{1} \mu^{2} \lambda^{2} \sigma=0
\end{gathered}
$$

Solving the algebraic equations given in (21) with the aid of Maple and Mathematica, we obtain the following results:

$$
c= \pm \sqrt{\frac{-p k^{2} \lambda-2 q}{\lambda}}, a_{0}=0, a_{1}= \pm \sqrt{\frac{q}{s \lambda}}, b_{1}= \pm \sqrt{\frac{q \lambda^{2} \sigma-q \mu^{2}}{s}} \text {, and } k=k
$$

Substituting these values into Eq. (16), we get the following solutions of Eq. (12).

Family 1.2.1:

$$
U(\xi) \pm \sqrt{\frac{q}{s \lambda}} \times\left(\frac{A_{1} \sqrt{\lambda} \cos (\sqrt{\lambda} \xi)-A_{2} \sqrt{\lambda} \sin (\sqrt{\lambda} \xi)}{A_{1} \sin (\sqrt{\lambda} \xi)+A_{2} \cos (\sqrt{\lambda} \xi)+\frac{\mu}{\lambda}}\right) \pm \sqrt{\frac{q \lambda^{2} \sigma-q \mu^{2}}{s}} \times\left(\frac{1}{A_{1} \sin (\sqrt{\lambda} \xi)+A_{2} \cos (\sqrt{\lambda} \xi)+\frac{\mu}{\lambda}}\right)
$$

where $\sigma=A_{1}^{2}+A_{2}^{2}$.

Family 1.2.2: When $A_{1}=0, A_{2} \neq 0$ and $\mu=0$ in Eq. (22), we obtain the solitary wave solution

$$
U(\xi)= \pm \sqrt{\frac{q}{s}} \tan (\sqrt{\lambda} \xi) \pm \sqrt{\frac{q \lambda^{2} \sigma-q \mu^{2}}{s}} \frac{1}{A_{2}} \sec (\sqrt{\lambda} \xi) .
$$

Family 1.2.3: When $A_{1} \neq 0, A_{2}=0$ and $\mu=0$ in Eq. (22), we obtain the solitary wave solution

$$
U(\xi)= \pm \sqrt{\frac{q}{s}} \cot (\sqrt{\lambda} \xi) \pm \sqrt{\frac{q \lambda^{2} \sigma-q \mu^{2}}{s}} \frac{1}{A_{2}} \operatorname{cosec}(\sqrt{\lambda} \xi) .
$$

Case 1.3: For $\lambda=0$, substituting Eq. (16) into Eq. (15) along with Eq. (2) and Eq.(8) yields a set of algebraic equations for $a_{0}, a_{1}, b_{1} \mu C, P$, and $K$ as follows:

$$
\begin{aligned}
& \varphi^{0}: 4 q a_{0} a_{1}^{2} \mu a_{2}-4 s a_{0}^{3} \mu^{2} a_{2}^{1}-s a_{0}^{3} a_{1}^{4}-4 q a_{0} \mu^{2} a_{2}^{2}+4 s a_{0}^{3} a_{1}^{2} \mu a_{2}-q a_{0} a_{1}^{4}=0 \\
& \varphi^{1}: 4 q a_{1}^{3} \mu a_{2}-8 p k^{2} a_{1} \lambda \mu^{2} a_{2}^{2}-3 s a_{0}^{2} a_{1}^{5}+12 s a_{0}^{2} a_{1}^{3} \mu a_{2}-2 c^{2} a_{1}^{5} \lambda-8 c^{2} a_{1} \lambda a_{2}^{2}+8 c^{2} a_{1}^{3} \lambda a_{2}+8 p k^{2} a_{1}^{3} \lambda \mu a_{2}-2 p k^{2} a_{1}^{5} \lambda \\
& -4 q a_{1} \mu^{2} a_{2}^{2}-12 s a_{0}^{2} a_{1} \mu^{2} a_{2}^{2}-q a_{1}^{5}=0 \\
& \varphi^{2}: 12 S a_{0} a_{1}^{4} \mu a_{2}-2 c^{2} b_{1} \mu^{2} a_{2}+p k^{2} b_{1} \mu a_{1}^{2}-2 p k^{2} b_{1} \mu^{2} a_{2}+6 s a_{0} b_{1}^{2} \mu a_{2}+2 s b_{1}^{3} \mu+c^{2} b_{1} \mu a_{1}^{2}-12 s a_{0} a_{1}^{2} \mu^{2} a_{2}^{2}-3 s a_{0} a_{1}^{6} \\
& -3 s a_{0} b_{1}^{2} a_{1}^{2}=0 \\
& \varphi^{3}: 6 s a_{1} b_{1}^{2} \mu a_{2}-2 p k^{2} a_{1}^{5}+8 p k^{2} a_{1}^{3} \mu a_{2}-8 c^{2} a_{1} \mu^{2} a_{2}^{2}-3 s a_{1}^{3} b_{1}^{2}-s a_{1}^{7}-4 s a_{1}^{3} \mu^{2} a_{2}^{2}-8 p k^{2} a_{1} \mu^{2} a_{2}^{2}+4 s a_{1}^{5} \mu a_{2} \\
& +8 c^{2} a_{1}^{3} \mu a_{2}-2 c^{2} a_{1}^{5}=0 \\
& \psi:-c^{2} b_{1} \lambda a_{1}^{4}-q b_{1} a_{1}^{4}-p k^{2} b_{1} a_{1}^{4} \lambda+12 s a_{0}^{2} b_{1} \mu a_{2} a_{1}^{2}+4 c^{2} b_{1} \mu^{3} a_{2}+4 q b_{1} a_{1}^{2} \mu a_{2}+4 c^{2} b_{1} \lambda \mu a_{2} a_{1}^{2}-4 s b_{1}^{3} \mu^{2} \\
& -12 s a_{0} b_{1}^{2} \mu^{2} a_{2}-4 p k^{2} b_{1} \lambda \mu^{2} a_{2}^{2}-3 s a_{0}^{2} b_{1} a_{1}^{4}-2 p k^{2} b_{1} \mu^{2} a_{1}^{2}+4 p k^{2} b_{1} \mu^{3} a_{2}-12 s a_{0}^{2} b_{1} \mu^{2} a_{2}^{2}-4 q b_{1} \mu^{2} a_{2}^{2} \\
& -4 c^{2} b_{1} \lambda \mu^{2} a_{2}^{2}+6 s a_{0} b_{1}^{2} \mu a_{1}^{2}+4 p k^{2} b_{1} \lambda a_{1}^{2} \mu a_{2}-2 c^{2} b_{1} \mu^{2} a_{1}^{2}=0 \\
& \psi \varphi:-12 c^{2} a_{1}^{3} \mu^{2} a_{2}+3 p k^{2} a_{1}^{5} \mu+6 s a_{1}^{3} b_{1}^{2} \mu+12 p k^{2} a_{1} \mu^{3} a_{2}^{2}+3 c^{2} a_{1}^{5} \mu-24 s a_{0} a_{1} b_{1} \mu^{2} a_{2}^{2}-6 s a_{0} a_{1}^{5} b_{1}-12 p k^{2} a_{1}^{3} \mu^{2} a_{2} \\
& +12 c^{2} a_{1} \mu^{3} a_{2}^{2}+24 s a_{0} a_{1}^{3} b_{1} \mu a_{2}-12 s a_{1} b_{1}^{2} \mu^{2} a_{2}=0 \\
& \psi \varphi^{2}:-3 s a_{1}^{6} b_{1}-2 c^{2} b_{1} a_{1}^{4}-12 s a_{1}^{2} b_{1} \mu^{2} a_{2}^{2}-8 c^{2} b_{1} \mu^{2} a_{2}^{2}+12 s a_{1}^{4} b_{1} \mu a_{2}-2 p k^{2} b_{1} a_{1}^{4}+8 c^{1} b_{1} \mu a_{2} a_{1}^{2}+8 p k^{2} b_{1} a_{1}^{2} \mu a_{2}- \\
& 8 p k^{2} b_{1} \mu^{2} a_{2}^{2}-s b_{1}^{3} a_{1}^{2}+2 s b_{1}^{3} \mu a_{2}=0
\end{aligned}
$$

Solving the algebraic equation with the aid of Maple or Mathematica, we obtain the following results:

$a_{0}=a_{0}, a_{1}= \pm \sqrt{2 \mu a_{2}}, b_{1}=0, k=k, c=c$

Substituting this into Eq. (17), we get the solution of Eq. (12) as follows:

Family: 1.3.1: 


$$
U(\xi)=a_{0} \pm \sqrt{2 \mu a_{2}}\left(\frac{\mu \xi+A_{1}}{\frac{\mu}{2} \xi^{2}+A_{1} \xi+A_{2}}\right)
$$

\subsection{The Density Dependent Fractional Diffusion Reaction Equation}

In this subsection we apply the $\left(G^{\prime} / G, 1 / G\right)$-expansion method to obtain exact solution of density dependent fractional diffusion reaction equation which can be given in the form:

$$
\frac{\partial^{\alpha} u(x, t)}{\partial t^{\alpha}}+k u(x, t) \frac{\partial^{\alpha} u(x, t)}{\partial x^{\alpha}}=D \frac{\partial^{2 \alpha} u(x, t)}{\partial x^{2 \alpha}}+a u(x, t)-b u^{2}(x, t)
$$

where, $t>0,0<\alpha<1$.

Introducing the following transformation:

$$
\xi=\frac{p x^{\alpha}}{\Gamma(1+\alpha)}-\frac{c t^{\alpha}}{\Gamma(1+\alpha)}, u(x, t)=U(\xi)
$$

where $p$ and $c$ are non-zero constants. Using traveling wave variable, equation (27) reduced into the following ODE for $u=$ $u(\xi)$ :

$$
D p^{2} U^{\prime \prime}+c U^{\prime}-k p U U^{\prime}+a U-b U^{2}=0
$$
form:

Balancing the highest order derivatives in linear and nonlinear terms, we get $N=1$. Therefore the solution of (29) is of the

$$
U(\xi)=a_{0}+a_{1} \varphi(\xi)+b_{1} \psi(\xi)
$$

Case 2.1: For $\lambda<0$, substituting equation (3.19) into equation (29) along with equation (2) and equation (4) yields a set of algebraic equation for $a_{0}, a_{1}, b_{1}, D, p, c, \lambda, \mu, \sigma$ as follows:

$$
\begin{aligned}
& \varphi^{0}:-k p a_{0} a_{1} \lambda \mu^{2}+c a_{1} \lambda \mu^{2}+b a_{0}^{2} \lambda^{2} \sigma-a a_{0} \lambda^{2} \sigma-k p a_{0} a_{1} \lambda^{3} \sigma-k p b_{1} \lambda^{2} a_{1} \mu+c a_{1} \lambda^{3} \sigma-D p^{2} b_{1} \lambda^{2} \mu-b b_{1}^{2} \lambda^{2}+b a_{0}^{2} \mu^{2} \\
& -a a_{0} \mu^{2}=0 \\
& \phi^{1}:-a a_{1} \lambda^{2} \sigma-2 D p^{2} a_{1} \lambda \mu^{2}+k p b_{1}^{2} \lambda^{2}-a a_{1} \mu^{2}-2 D p^{2} a_{1} \lambda^{3} \sigma+2 b a_{0} a_{1} \lambda^{2} \sigma+2 b a_{0} a_{1} \mu^{2}-k p a_{1}^{2} \mu^{2} \lambda \\
& -\boldsymbol{k p a}_{1}^{2} \lambda^{3} \sigma=0 \\
& \phi^{2}:-k p a_{0} a_{1} \mu^{2}-k p b_{1} \lambda a_{1} \mu+b a_{1}^{2} \mu^{2}-k p a_{0} a_{1} \lambda^{2} \sigma+c a_{1} \lambda^{2} \sigma-b b_{1}^{2} \lambda+c a_{1} \mu^{2}-D p^{2} b_{1} \lambda \mu+b a_{1}^{2} \lambda^{2} \sigma=0 \\
& \phi^{3}:-k p a_{1}^{2} \lambda^{2} \sigma+k p b_{1} \lambda-k p a_{1}^{2} \mu^{2}-2 D p^{2} a_{1} \lambda^{2} \sigma-2 D p^{2} a_{1} \mu^{2}=0 \\
& \psi:-a b_{1} \mu^{2}-c a_{1} \mu \lambda^{2} \sigma+k p a_{0} a_{1} \mu^{3}-D p^{2} b_{1} \lambda^{3} \sigma+2 b a_{0} b_{1} \lambda^{2} \sigma-c a_{1} \mu^{3}+2 b a_{0} b_{1} \mu^{2}+k p b_{1} a_{1} \lambda \mu^{2} \\
& +D p^{2} b_{1} \lambda \mu^{2}-a b_{1} \lambda^{2} \sigma+k p a_{0} a_{1} \mu \lambda^{2} \sigma+2 b b_{1}^{2} \lambda \mu-k p b_{1} a_{1} \lambda^{3} \sigma=0 \\
& \psi \phi: c b_{1} \lambda^{2} \sigma+3 D p^{2} a_{1} \mu^{3}-2 k p b_{1}^{2} \lambda \mu+3 D p^{2} a_{1} \mu \lambda^{2} \sigma-k p a_{0} b_{1} \lambda^{2} \sigma-k p a_{0} b_{1} \mu^{2}+c b_{1} \mu^{2}+2 b a_{1} b_{1} \mu^{2} \\
& +\boldsymbol{k p a _ { 1 } ^ { 2 }} \mu^{3}+\boldsymbol{k p a _ { 1 } ^ { 2 }} \mu \lambda^{2} \sigma+2 b a_{1} b_{1} \lambda^{2} \sigma=0 \\
& \psi \phi^{2}:-2 k p a_{1} b_{1} \mu^{2}-2 D p^{2} b_{1} \mu^{2}-2 D p^{2} b_{1} \lambda^{2} \sigma-2 k p a_{1} b_{1} \lambda^{2} \sigma=0
\end{aligned}
$$

Solving the algebraic equation (31) by using the software Maple or Mathematica, we obtain the following results:

$$
\begin{gathered}
a_{0}=\frac{a}{2 b}, a_{1}=\frac{ \pm a \sqrt{-\frac{1}{4 \lambda}}}{b}, b_{1}=\frac{ \pm a \sqrt{\mu^{2}+\lambda^{2} \sigma}}{2 \lambda b}, c= \pm \frac{\left(4 b^{2} D+k^{2} a\right) a}{8 b^{2} D \lambda \sqrt{-\frac{1}{4 \lambda}}} \\
\text { and } p= \pm \frac{a k \sqrt{-\frac{1}{4 \lambda}}}{b D} .
\end{gathered}
$$

$$
\begin{aligned}
\boldsymbol{U}(\xi) & =\frac{\boldsymbol{a}}{2 \boldsymbol{b}} \pm \frac{\boldsymbol{a} \sqrt{-\frac{1}{4 \lambda}}}{\boldsymbol{b}} \frac{A_{1} \sqrt{-\lambda} \cosh (\sqrt{-\lambda} \xi)+A_{2} \sqrt{-\lambda} \sinh (\sqrt{-\lambda} \xi)}{A_{1} \sinh (\sqrt{-\lambda} \xi)+A_{2} \cosh (\sqrt{-\lambda} \xi)+\frac{\mu}{\lambda}} \\
& \pm \frac{\boldsymbol{a} \sqrt{\mu^{2}+\lambda^{2} \sigma}}{2 \lambda \boldsymbol{b}} \times \frac{1}{A_{1} \sinh (\sqrt{-\lambda} \xi)+A_{2} \cosh (\sqrt{-\lambda} \xi)+\frac{\mu}{\lambda}}
\end{aligned}
$$

Substituting this into equation (30) we get the solution of (29) as follows:

Family 2.1.1:

where, $\sigma=A_{1}^{2}-A_{2}^{2}$.

Family 2.1.2: If $A_{1}=0, A_{2} \neq 0$ and $\mu=0$ in (32), we get the solitary wave solution 


$$
\boldsymbol{U}(\xi)=\frac{\boldsymbol{a}}{2 \boldsymbol{b}} \pm \frac{\boldsymbol{a} \sqrt{-\frac{1}{4 \lambda}} \cdot \sqrt{\lambda} \tanh (\sqrt{-\lambda} \xi)}{\boldsymbol{b}} \pm \frac{\boldsymbol{a} \sqrt{\mu^{2}+\lambda^{2} \sigma}}{2 \lambda \boldsymbol{b}} \cdot \frac{1}{\boldsymbol{A}_{2}} \sec \boldsymbol{h}(\sqrt{-\lambda} \xi)
$$

Family 2.1.13: If $A_{1} \neq 0, A_{2}=0$ and $\mu=0$ in (32), we get the solitary wave solution

$$
\begin{aligned}
& \phi^{0}:-D p^{2} b_{1} \lambda^{2} \mu-b a_{0}^{2} \lambda^{2} \sigma-k p b_{1} \lambda^{2} a_{1} \mu-b b_{1}^{2} \lambda^{2}-c a_{1} \lambda^{3} \sigma+c a_{1} \lambda \mu^{2}+a a_{0} \lambda^{2} \sigma+b a_{0}^{2} \mu^{2}
\end{aligned}
$$

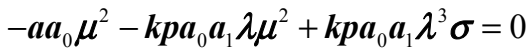

$$
\begin{aligned}
& \phi^{1}: \boldsymbol{k p a _ { 1 } ^ { 2 }} \lambda^{3} \sigma+2 b a_{0} a_{1} \mu^{2}+a a_{1} \lambda^{2} \sigma-a a_{0} \mu^{2}-2 b a_{0} a_{1} \lambda^{2} \sigma-2 D p^{2} a_{1} \lambda \mu^{2}+D p^{2} a_{1} \lambda^{3} \sigma \\
& +\boldsymbol{k p} \boldsymbol{b}_{1}^{2} \lambda^{2}-\boldsymbol{k p a _ { 1 } ^ { 2 }} \lambda \mu^{2}=0 \\
& \phi^{2}=c a_{1} \mu^{2}+b a_{1}^{2} \mu^{2}-D p^{2} b_{1} \lambda \mu-b b_{1}^{2} \lambda-k p a_{0} a_{1} \mu^{2}-k p b_{1} \lambda a_{1} \mu+b a_{1}^{2} \lambda^{2} \sigma+k p a_{0} a_{1} \lambda^{2} \sigma-c a_{1} \lambda^{2} \sigma=0 \\
& \phi^{3}: \boldsymbol{k p a}_{1}^{2} \lambda^{2} \sigma-2 D p^{2} a_{1} \mu^{2}+\boldsymbol{k p b}_{1}^{2} \lambda+2 D p^{2} a_{1} \lambda^{2} \sigma-k p a_{1}^{2} \mu^{2}=0 \\
& \psi:-C a_{1} \mu^{3}+C a_{1} \mu \lambda^{2} \sigma+K P a_{0} a_{1} \mu^{3}+D P^{2} b_{1} \lambda \mu^{2}+2 b a_{0} b_{1} \mu^{2}+D P^{2} b_{1} \lambda^{3} \sigma+K P b_{1} a_{1} \lambda^{3} \sigma+K P b_{1} a_{1} \lambda \mu^{2}-K P a_{0} a_{1} \mu \lambda^{2} \sigma \\
& -2 b a_{0} b_{1} \lambda^{2} \sigma+2 b b_{1}^{2} \lambda \mu-a b_{1} \mu^{2}+a b_{1} \mu^{2}+a b_{1} \lambda^{2} \sigma=0 \\
& \psi \varphi:-K P a_{0} b_{1} \mu^{2}+C b_{1} \mu^{2}+K P a_{1}^{2} \mu^{3}-C b_{1} \lambda^{2} \sigma+K P a_{0} b_{1} \lambda^{2} \sigma-2 b a_{1} b_{1} \lambda^{2} \sigma-2 K P b_{1}^{2} \lambda \mu+2 b a_{1} b_{1} \mu^{2}+3 D P^{2} a_{1} \mu^{3} \\
& -K P a_{1}^{2} \mu \lambda^{2} \sigma-3 D P^{2} a_{1} \mu \lambda^{2} \sigma=0 \\
& \psi \varphi^{2}: 2 D P^{2} b_{1} \lambda^{2} \sigma-2 K P a_{1} b_{1} \mu^{2}-2 D P^{2} b_{1} \mu^{2}+2 K P a_{1} b_{1} \lambda^{2} \sigma=0
\end{aligned}
$$

Solving the algebraic equation (35) by using the software Maple or Mathematica, we obtain the following results:

$$
\begin{gathered}
a_{0}=\frac{a}{2 b}, a_{1}= \pm \frac{a \sqrt{-\frac{1}{4 \lambda}}}{b}, b_{1}= \pm \frac{a \sqrt{\mu^{2}-\lambda^{2} \sigma}}{2 \lambda b} \\
C= \pm \frac{\left(4 b^{2} D+K^{2} \alpha\right) a}{8 b^{2} D \lambda \cdot \sqrt{-\frac{1}{4 \lambda}}}, \text { and } P=\frac{a K \sqrt{-\frac{1}{4 \lambda}}}{b D}
\end{gathered}
$$

Substituting this into Eq. (30), we get the solution of (29) as follows:

Family 2.2.1:

$$
\begin{gathered}
U(\xi)=\frac{a}{2 b} \pm \frac{a \sqrt{-\frac{1}{4 \lambda}}}{b} \times \frac{A_{1} \sqrt{\lambda} \cos (\sqrt{\lambda} \xi)+A_{2} \sqrt{\lambda} \sin (\sqrt{\lambda} \xi)}{A_{1} \sin (\sqrt{\lambda} \xi)+A_{2} \cos (\sqrt{\lambda} \xi)+\frac{\mu}{\lambda}} \\
\pm \frac{a \sqrt{\mu^{2}-\lambda^{2} \sigma}}{2 \lambda b} \times \frac{1}{A_{1} \sin (\sqrt{\lambda} \xi)+A_{2} \cos (\sqrt{\lambda} \xi)+\frac{\mu}{\lambda}}
\end{gathered}
$$

where $\sigma=A_{1}^{2}+A_{2}^{2}$.

Family 2.2.2: If $A_{1}=0, A_{2} \neq 0$ and $\mu=0$ in (36), we get the solitary wave solution

$$
U(\xi)=\frac{a}{2 b} \pm \frac{a \sqrt{-\frac{1}{4 \lambda}}}{b} \sqrt{\lambda} \tan (\sqrt{\lambda} \xi) \pm \frac{a \sqrt{\mu^{2}-\lambda^{2} \sigma}}{2 \lambda b} \frac{1}{A_{2}} \sec (\sqrt{\lambda} \xi)
$$

Family 2.2.3: If $A_{1} \neq 0, A_{2}=0$ and $\mu=0$ in (36), we get the Solitary wave solution

$$
U(\xi)=\frac{a}{2 b} \pm \frac{a \sqrt{-\frac{1}{4 \lambda}}}{b} \sqrt{\lambda} \cot (\sqrt{\lambda} \xi) \pm \frac{a \sqrt{\mu^{2}-\lambda^{2} \sigma}}{2 \lambda b} \frac{1}{A_{2}} \operatorname{cosec}(\sqrt{\lambda} \xi)
$$

Case 2.3: For $\lambda=0$, substituting Eq. (30) into Eq. (29) along with Eq. (2) and Eq. (7) yields a set of algebraic equations for $a_{0}, a_{1}, b_{1}, D, P, C, \lambda, \mu, C, P$ as follows:

$$
\begin{gathered}
\varphi^{0}:-C a_{1}^{3} \lambda-b a_{0}^{2} a_{1}^{2}-2 a a_{0} \mu a_{2}-2 K P a_{0} a_{1} \lambda \mu a_{2}+a a_{0} a_{1}^{2}+2 C a_{1} \lambda \mu a_{2}+K P a_{0} a_{1}^{3} \lambda+2 b a_{0}^{2} \mu a_{2}=0 \\
\varphi^{1}:-2 a a_{1} \mu a_{2}-2 K P a_{1}^{2} \lambda \mu a_{2}-2 b a_{0} a_{1}^{3}+2 D P^{2} a_{1}^{3} \lambda-4 D P^{2} a_{1} \lambda \mu a_{2}+4 b a_{0} a_{1} \mu a_{2}+K P a_{1}^{4} \lambda+a a_{1}^{3}=0
\end{gathered}
$$




$$
\begin{gathered}
\varphi^{2}: K P a_{0} a_{1}^{3}-K P b_{1} a_{1} \mu-b b_{1}^{2}+2 b a_{1}^{2} \mu a_{2}+2 C a_{1} \mu a_{2}-D P^{2} b_{1} \mu-2 K P a_{0} a_{1} \mu a_{2}-b a_{1}^{4}-C a_{1}^{3}=0 \\
\varphi^{3}: 2 D P^{2} a_{1}^{3}+K P b_{1}^{2}-4 D P^{2} a_{1} \mu a_{2}+K P a_{1}^{4}-2 K P a_{1}^{2} \mu a_{2}=0 \\
\psi: 2 K P b_{1} a_{1} \mu^{2}-K P a_{0} a_{1}^{3} \mu+2 K P a_{0} a_{1} \mu^{2} a_{2}+C a_{1}^{3} \mu+2 D P^{2} b_{1} \mu^{2}+a b_{1} a_{1}^{2}+2 b b_{1}^{2} \mu+4 b a_{0} b_{1} \mu a_{2}-2 C a_{1} \mu^{2} a_{2} \\
+K P b_{1} a_{1}^{3} \lambda+D P^{2} b_{1} \lambda a_{1}^{2}-2 a_{1} \mu a_{2}-2 D P^{2} b_{1} \lambda \mu a_{2}-2 b a_{0} b_{1} a_{1}^{2}-2 K P b_{1} a_{1} \lambda \mu a_{2}=0 \\
\varphi \psi: 6 D P^{2} a_{1} \mu^{2} a_{2}-3 D P^{2} a_{1}^{3} \mu-K P a_{1}^{4} \mu+4 b a_{1} b_{1} \mu a_{2}+2 K P a_{1}^{2} \mu^{2} a_{2}-2 b a_{1}^{3} b_{1}+2 C b_{1} \mu a_{2}+2 K P b_{1}^{2} \mu-2 K P a_{0} b_{1} \mu a_{2} \\
+K P a_{0} b_{1} a_{1}^{2}-C b_{1} a_{1}^{2}=0 \\
\psi \varphi^{2}: 2 K P a_{1}^{3} b_{1}+2 D P^{2} b_{1} a_{1}^{2}-4 K P a_{1} b_{1} \mu a_{2}-4 D P^{2} b_{1} \mu a_{2}=0
\end{gathered}
$$

Solving the algebraic equation (39) by using the software Maple or Mathematica, we obtain the following results:

$$
a_{0}=a_{0}, a_{1}= \pm \sqrt{2 \mu a_{2}}, b_{1}=0, C=C \text { and } P=P .
$$

Substituting this into Eq. (30), we get the solution of (29) as follows:

Family 2.3.1:

$$
U(\xi)=a_{0} \pm \sqrt{2 \mu a_{2}} \times\left(\frac{\mu \xi+A_{1}}{\frac{\mu}{2} \xi^{2}+A_{1} \xi+A_{2}}\right) .
$$

\section{Conclusion}

In this article, the two variable $\left(G^{\prime} / G, 1 / G\right)$-expansion method is used to obtain further general and some new as well as some known solutions of the well-known fractional generalized reaction Duffing model and density dependent fractional diffusion reaction equation. If we take the special value of two parameters $A_{1}$ and $A_{2}$, we get the solitary wave solutions. When $\mu=0$ and $b_{i}=0$ in (1) and (11) respectively, the two variable $\left(G^{\prime} / G, 1 / G\right)$-expansion method transferred to the original $\left(G^{\prime} / G\right)$-expansion method. Thus, the two variable $\left(G^{\prime} / G, 1 / G\right)$-expansion is an extension of the $\left(G^{\prime} / G\right)$-expansion method. The two variable $\left(G^{\prime} / G, 1 / G\right)$-expansion method applied in this article is more efficient and more general than the original $\left(G^{\prime} / G\right)$ expansion method.

\section{References}

[1] R. Hirota, Exact solutions of the Kdv equation for multiple collisions of solutions, Physical Review Letters 27(1) 11921194, 1971.

[2] M. R Miura, Backlund Transformation, Springer, Berlin, Germany, 1978.

[3] C. Rogers and W. F. Shadwick, Backlund Transformation, PP. Academic Press-New-York, NY, USA, 1982.

[4] M. J Ablowitz and P. A. Clarkson, Soliton, Nonlinear Evolution equations and Inverse Scattering, vol.149 of London Mathematical Society Lecture Note Series, Cambridge University Press, New York, NY, USA, 1991.

[5] Y. Chen and Q. Wang, Extended Jacobi elliptical function rational expression method and abundant families of Jacobi elliptic function solution to $(1+1)$-dimensional dispersive long wave equation, Chaos, Solitons and Fractals, 24(3), 745-757, 2005 .
[6] S. Liu, Z. Fu and Q. Zhao, Jacobi elliptic function expression method and periodic wave solutions of nonlinear wave equations, Physics Letters A, 289(1-2), 69-74, 2001.

[7] D. LU, Jacobi elliptic function solutions for two variable Boussinesq equations, chaos, solutions and Fractals, 24(5), 1973-1385, 2005.

[8] J. Weiss, M. Tabor and Gcarnevale, The painleve property for partial differential equations, Journal of Mathematical Physics, 24(3), 522-526, 1983

[9] N. A Kudryashov, Exact soliton Solution of the generalized evolution equation of wave dynamics, Journal of Mathematics and Mechanics, 52(1), 361-365, 1988.

[10] N. A Kudryashov, Exact soliton Solution of the generalized Kuramoto-Sivashinsky equation, Physics Letter A, 147(5-6), 287-275, 1991.

[11] N. A Kudryashov, on types of the nonlinear nonintegrableequations with exact Solutions, Physics Letter A, 155(4-5), 269-275, 1991.

[12] N. A Abdou, The extended tanh method and its application for solving nonlinear physical models, Applied Mathematics and Computations, 190(1), 988-996, 2007.

[13] E. Fan, Extended tanh method and its application for solving nonlinear equation, Physics Letter A, 277(4-5), 212-218, 2000.

[14] S. Zhang and T. C. Xia, A further improved function method exactly solving the $(2+1)$-dimensional dispersion long wave equations, Applied Mathematics E-Notes, 8(1), 58-66, 2008.

[15] E. Yusufogla and A. Bekir, Exact solutions of coupled nonlinear Klien-Gordon equation, Mathematical and Computer Modeling, 48(11-12), 1694-1700, 2008.

[16] J. H. He and X. H. WV, Exp-function method for nonlinear wave equations, Chaos, Solitons and Fractals, 30(3), 700-708, 2006 .

[17] E. Yusufogla, New solitary for the MBBM equation using Expfunction method, Physics Letter A, 372, 442-446, 2008.

[18] S. Zhang, Application of Exp-function method to highdimensional nonlinear evolution equation, Chaos, Soliton and Fractals, 38(1), 270-276, 2008.

[19] A. Bekir, the Exp-function for Ostovsky equation, International Journal of Nonlinear Science and Numerical Simulation, 10(1), 735-739, 2009.

[20] A. Bekir, Application of the Exp-function method for nonlinear differential difference equations, Applied Mathematics and Computation, 215(11), 4049-4053, 2010. 
[21] Nahar H, Abdullah FA, Akbar MA, New traveling wave solution of the higher dimensional nonlinear PDE by Expfunction method, "Journal of Applied Mathematics, Vol. 2012, 1603-1610, 2012.

[22] Akbar MA, Ali NHM, New solitary and periodic solutions of nonlinear evolution equation by Exp- function method, World Applied Sciences Journal, 17(12),1603-1610, 2012.

[23] M. Wang, X. Li and J. Zhang, The (G'/G)-expression method and traveling wave solutions of nonlinear evolution equations in mathematical physics, Physics Letter A, 372(4), 417-423, 2008.

[24] S. Zhang, J. L. Tong and W. Wang, A generalized (G'/G)expression method for the $\mathrm{mKdV}$ equation with variable coefficients, Physics Letter A, 372, 2254-2257, 2008.

[25] E. M. E Zayed and K. A. Gepreel, $\left(\mathrm{G}^{\prime} / \mathrm{G}\right)$-expression for finding traveling wave solutions of the nonlinear PDE in the mathematical physics, Journal of Mathematical Physics, 50(1), ID 13502, 2009.

[26] E. M. E Zayed, The $\left(\mathrm{G}^{\prime} / \mathrm{G}\right)$-expression method and its application to some nonlinear evolution equations in mathematical physics, Journal of Mathematical Physics, 30(12), 89-103, 2009.

[27] A Bekir, Application of the $\left(\mathrm{G}^{\prime} / \mathrm{G}\right)$-expression method for nonlinear evolution equations, Communications in Nonlinear Science and Numerical Simulation, 372(19), 3400-3406, 2008.

[28] B. Ayham and A. Bekir, The $\left(\mathrm{G}^{\prime} / \mathrm{G}\right)$-expression method for the nonlinear lattice equations, Communications in Nonlinear Science and Numerical Simulation, 17(9), 3490-3498, 2012.

[29] N. A Kudryshov, A note on the (G'/G)-expression method, Applied Mathematics and Computation, 217(4), 1755-1758, 2010.

[30] I. Aslam, A note on the $\left(\mathrm{G}^{\prime} / \mathrm{G}\right)$-expression method again, Applied Mathematics and Computation, 217(2), 937-938, 2010.

[31] Akbar MA, Ali NHM, Zayed EME, A generalized and improved $\left(\mathrm{G}^{\prime} / \mathrm{G}\right)$-expansion method for nonlinear evolution equation, Math. Prob. Engr., Vol. 2012, 1-22, 2012.

[32] Akbar MA, Ali NHM, Zayed EME, Abundant exact traveling wave solutions of the generalized Bretherton equation via the improved ( $\left.\mathrm{G}^{\prime} / \mathrm{G}\right)$-expansion method, Communication in Theoretical Physics, 57(2), 173-178, 2012.

[33] L. X. Li, E. Q. Li and M. L. Wang, the (G'/G, 1/G)-expansion method and its application to travelling wave solutions of the Zakharov equation, Applied Mathematics B, 25(4), 454-462, 2010 .

[34] E. M. E. Zayed and M. A. M. Abdelaziz, The two variable $\left(\mathrm{G}^{\prime} / \mathrm{G}, 1 / \mathrm{G}\right)$-expansion method for solving the nonlinear KdVmKdV equation, Math. Prob. Engr., 2012, ID 725061, 14 pages.

[35] F. Tascam and A. Bekir, Applications of the first integral method to the nonlinear evolution equation, Chinese Physics B, 19, ID 080201, 2010. 